BULLETIN OF THE

AMERICAN MATHEMATICAL SOCIETY

Volume 78, Number 4, July 1972

\title{
HOMOTOPY GROUPS OF FINITE $H$-SPACES
}

BY JOHN R. HARPER ${ }^{1}$

\author{
Communicated by Morton Curtis, December 2, 1971
}

In this announcement we present results about the homotopy groups of $H$-spaces having the homotopy type of finite $\mathrm{CW}$-complexes. We call such spaces finite $H$-spaces. We always assume our spaces are connected. In the sequel we always use $X$ to denote a finite $H$-space. In some statements we refer to a direct sum of cyclic groups. We do not rule out the case that the sum is zero.

Let $\tilde{X}$ be the fibre of the canonical map

$$
X \rightarrow K\left(\Pi_{1}(X), 1\right) .
$$

It is well known that this "universal covering space" $\tilde{X}$ is a finite $H$-space.

THEOREM 1. $\Pi_{4}(X)$ is a direct sum of groups of order $2, \operatorname{dim} \Pi_{4}(X)^{\prime}$ $=\operatorname{dim} \operatorname{ker} \mathrm{Sq}^{2}: H^{3}\left(\tilde{X}: Z_{2}\right) \rightarrow H^{5}\left(\tilde{X}: Z_{2}\right)$.

Proof. Since $\tilde{X}$ is a finite $H$-space, it suffices to work with simply connected $X$. We use the exact sequence of J. H. C. Whitehead,

$$
\rightarrow H_{n+1}(X: Z) \stackrel{v_{n}}{\rightarrow} \Gamma_{n}(X) \stackrel{\lambda_{n}}{\rightarrow} \Pi_{n}(X) \stackrel{h_{n}}{\rightarrow} H_{n}(X: Z) \rightarrow .
$$

Results of Browder [3] and Hilton [7] give $\Gamma_{4}(X) \cong H_{3}\left(X: Z_{2}\right)$. Browder's Theorem 6.1 of [3] yields

LEMMA 2. Let $X$ be simply connected, then $H_{4}(X: Z)=0$.

From [7] we obtain $v_{4}$ as the composite

$$
H_{5}(X: Z) \stackrel{r}{\rightarrow} H_{5}\left(X: Z_{2}\right) \stackrel{\mathrm{Sq}^{2}}{\rightarrow} H_{3}\left(X: Z_{2}\right)
$$

where $r$ is reduction mod 2. The theorem follows.

We remark that if $X$ is simply connected and $H_{*}(\Omega X: Z)$ torsion free, then Theorem 1 is contained in Bott-Samelson [2].

For the remainder of this paper we assume that $X$ is simply connected and $H_{*}(\Omega X: Z)$ is torsion free. We identify $\Gamma_{4}(X), H_{3}\left(X: Z_{2}\right)$ and $\Pi_{3}(X) \otimes Z_{2}$, and continue to use $v_{4}$. For $k \geqq 3, \eta_{k}: S^{k+1} \rightarrow S^{k}$ is the essential map.

THEOREM 3. The following sequence is exact,

$$
0 \rightarrow \Pi_{4}(X) \stackrel{\eta_{*}^{*}}{\rightarrow} \Pi_{5}(X) \stackrel{\underline{m}_{5}}{\rightarrow} H_{5}(X: Z) \stackrel{v_{4}}{\rightarrow} \Pi_{3}(X) \otimes Z_{2} \stackrel{\eta_{3}^{*}}{\rightarrow} \Pi_{4}(X) \rightarrow 0,
$$

with $\operatorname{ker} h_{5}=$ tors $\Pi_{5}(X)$, the torsion subgroup of $\Pi_{5}(X)$.

AMS 1970 subject classifications. Primary 55D45, 55E99; Secondary 57F20, 57F25.

${ }^{1}$ Research supported by grants from NSF and CAPES (Brasil). 
OUTLINE OF PROOF. In the appropriate segment of the Whitehead sequence, use [7] to show $\lambda_{5} \Gamma_{5}(X) \cong \Pi_{4}(X)$. From the Cartan-Serre Theorem [9] we have ker $h_{5} \subset$ tors $\Pi_{5}$. To prove the opposite inclusion we first use a theorem of Clark [6] which yields the fact that the $p$-torsion of $H_{*}(X: Z)$ is of order at most $p$. Applying a theorem of Browder [4] gives $H_{5}(X: Z)=F \oplus T$ where $F$ is free and $T$ is a direct sum of cyclic groups of order 2 . We then use arguments involving the Serre spectral sequence to show that if $h_{5}\left(\right.$ tors $\left.\Pi_{5}(X)\right) \neq 0$ then $H_{*}(\Omega X: Z)$ has torsion. The remaining details are straightforward.

Further use of the Whitehead sequence and [7] yields

THEOREM 4. Let $p$ be a prime. If $p \geqq 5$, then $\Pi_{6}(X)$ is p-torsion free. The 3-torsion is of order at most 3 and the 2-torsion of order at most 4 .

More detailed information can be obtained by means of the MasseyPeterson spectral sequence [8] and its extensions to odd primes [5]. The hypotheses for the use of the spectral sequence include $H^{*}\left(X: Z_{p}\right)=\bigcup(M)$ as algebras over the Steenrod algebra. Many $H$-spaces satisfy this but I know of no general result for finite $H$-spaces. However, if one can prove that $H^{*}\left(X: Z_{p}\right)$ satisfies this condition through a range of dimensions, then the spectral sequence can be used to calculate homotopy groups in a slightly smaller range. Via this technique, we obtain the following results:

THEOREM 5. Let $p$ be a prime. Then $\Pi_{n}(X)$ is p-torsion free for $n<2 p$ and the p-torsion of $\Pi_{2 p}(X)$ is of order at most $p$. Furthermore, for odd primes, $\operatorname{dim} \Pi_{2 p}(X) \otimes Z_{p}=\operatorname{dim} \operatorname{ker} P^{1}: H^{3}\left(X: Z_{p}\right) \rightarrow H^{2 p+1}\left(X: Z_{p}\right)$.

Our remaining results require a hypothesis in addition to those already carried. Equivalent forms are given in the next statement.

Proposition 6. The following statements are equivalent:

(a) $H^{5}\left(X: Z_{2}\right)=\mathrm{Sq}^{2} H^{3}\left(X: Z_{2}\right)$;

(b) $\operatorname{im} h_{5}=2 H_{5}(X: Z)$;

(c) the 5-skeleton $X^{5}$ is a bouquet of types $S^{3}$ and $S^{3} \cup_{\eta_{3}} e^{5}$;

(d) $\operatorname{dim} \Pi_{4}(X)=\operatorname{dim} H_{3}\left(X: Z_{2}\right)-\operatorname{dim} H_{5}\left(X: Z_{2}\right)$.

We conjecture that these statements are true in general.

Theorem 7. Assume the statements of Proposition 6 are true. Then

$$
\operatorname{dim} \Pi_{6} \otimes Z_{2} \leqq \operatorname{dim}\left[\left(\mathrm{ker} \mathrm{Sq}{ }^{3} \cap \mathrm{ker} \mathrm{Sq}^{4} \mathrm{Sq}^{2}\right) H^{3}\left(X: Z_{2}\right)\right]
$$

the torsion subgroup of $\Pi_{7}(X)$ is a direct sum of cyclic groups of order 2 .

The statement for $\Pi_{6}$ means "the dimension of the intersection of the kernals of the listed cohomology operations when applied to $H^{3}\left(X: Z_{2}\right)$." The proofs of Theorem 5 and the part about $\Pi_{7}$ essentially involve only 
the calculation of $E_{2}$ of the spectral sequence. The part about $\Pi_{6}$ involves a differential.

In summary, we list in tabular form the structure of the first seven homotopy groups. The table is for $H$-spaces $X$ such that $H^{*}\left(\Omega \tilde{X}: Z_{2}\right)$ is torsion free and $\tilde{X}$ satisfies Proposition 6. We use $F$ to mean a free group and $T_{n}$ a direct sum of cyclic groups of order $n$. Assuming Proposition 6 allows us to improve Theorems 1 and 3.

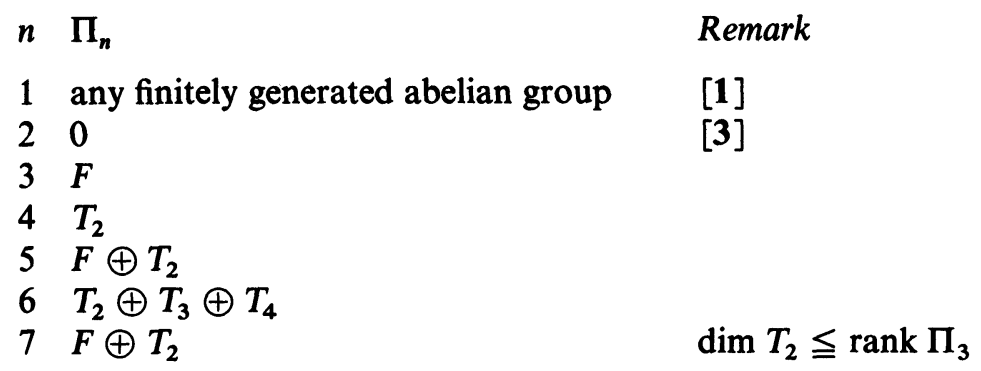

\section{REFERENCES}

1. A. Borel, Sur l'homologie et la cohomologie des groups de Lie compacts connexes, Amer. J. Math. 76 (1954), 273-342. MR 16, 219.

2. R. Bott and $\mathrm{H}$. Samelson, Zpplications of the theory of Morse to symmetric spaces, Amer. J. Math. 80 (1958), 964-1029. MR 21 \# 4430.

3. W. Browder, Torsion in H-spaces, Ann. of Math. (2) 74 (1961), 24-51. MR 23 \# A2201.

4., Higher torsion in H-spaces, Trans. Amer. Math. Soc. 108 (1963), 353-375. MR 27 \# 5260.

5. A. Bousfield and D. Kan, The homotopy spectral sequence of a space with coefficients in a ring, Topology 11 (1972), 79-106.

6. A. Clark, Hopf algebras over Dedekind domains and torsion in H-spaces, Pacific J. Math. 15 (1965), 419-426. MR 32 \#6453.

7. P. J. Hilton, Calculations of the homotopy groups of $A_{n}^{2}$-polyhedra. II, Quart. J. Math. Oxford Ser. (2) 2 (1951), 228-240. MR 13, 267.

8. W. S. Massey and F. P. Peterson, The mod 2 cohomology structure of certain fibre spaces, Mem. Amer. Math. Soc. No. 74 (1967). MR 37 \#2226.

9. J. W. Milnor and J. C. Moore, On the structure of Hopf algebras, Ann. of Math. (2) 81 (1965), 211-264. MR 30 \# 4259.

Pontificia Universidade Catolica, Rio de Janeiro, Brazil

Department of Mathematics, University of Rochester, Rochester, NeW York 14627 (Current address of John R. Harper) 